\title{
The Analysis of Analog Circuits Fault Diagnosis Methods
}

\author{
Zhiqiang Zhang ${ }^{1, a^{*}}$ and Aihua Zhang ${ }^{1, b}$ \\ ${ }^{1}$ College of Engineering, Bohai University, Jinzhou, China \\ aJsxinxi_zzq@163.com
}

Keywords: Analog circuit; Fault diagnosis; Diagnosis methods

\begin{abstract}
Focusing on the requirement of analog circuit fault and diagnosis, authors summarize the analog circuit fault classifier and analysis the corresponding analog circuit fault diagnosis methods based reviewing a great number of literatures. The fault diagnosis strategies are discussed from the two fields, one is the traditional fault diagnosis methods, and the other one is modern intelligent fault diagnosis method.
\end{abstract}

\section{Introduction}

Analog circuit fault diagnosis has much uncertainty and difficulty. Now, there are lots of fault diagnosis methods are disscussed in analog circuit fault diagnosis, such as based on data driven theory[1-3], based on fault dictionary idea, based on parameter identification idea and fault verification. Another kind of fault diagnosis method such as based on neural network, fuzzy theory, wavelet transform, genetic algorithm theory are all payed attention to. This article will focus on the present fault diagnosis methods, to give out the corresponding comparative analysis.

\section{Traditional Analog Circuit Fault Diagnosis Method}

Fault Dictionary Method. What's called fault dictionary is that all the fault types could be found in the fault dictionary. In the real diagnosis, once the fault appeared, the accurate fault diagnosis can be done [2]. The fault dictionary is supposed to be completed before failure. In order to build up the fault dictionary, first the fault types should be reserved, reoccupy circuit analysis program, circuit simulation. Calculates the node voltage, build up the fault mode, last modified into fault dictionary, and stored for retrieval in the computer again. Circuit under test under the control of the automatic test system, choosing the right incentives and the necessary test point, the node voltage measured to the electronic computer. Computer input test data and the retrieved from a dictionary stored data according to certain criteria, such as the rule of minimum bias squares, comparison, judgment, and display ${ }^{[3]}$. The work flow chart shown in Fig. 1

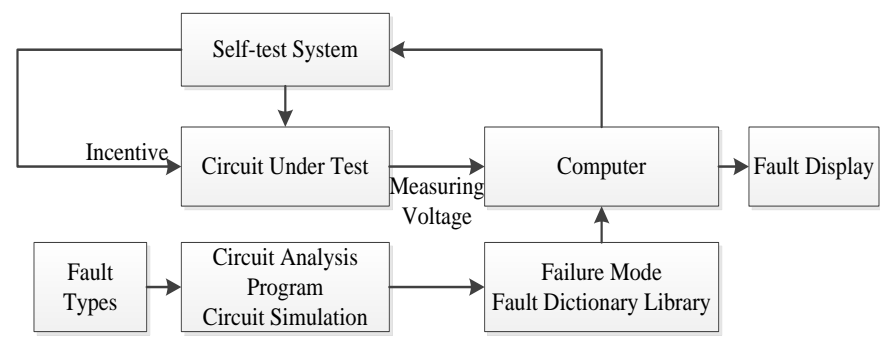

Figure 1. The work flow chart of fault dictionary method

Parameter Identification Method. Parameter identification technology is based on known topology relationship of analog circuit, input and output response, identify all components in circuit parameters, 
and then identify the parameters of the compared with allowed tolerance range of components, beyond the range of components for the fault components. The work flow chart is shown in Fig. 2.

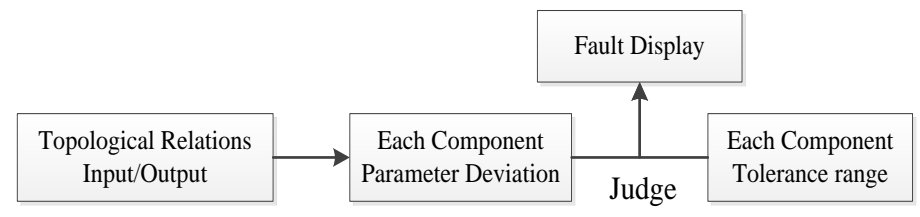

Figure 2. Work flow chart of parameter identification method

Fault Verification Method. Fault confirmed is the technology which is advance speculation in the circuit fault element combination, and calculates the combination, results, and fault phase, whether this combination is correct. The work flow chart is shown in Fig. 3.

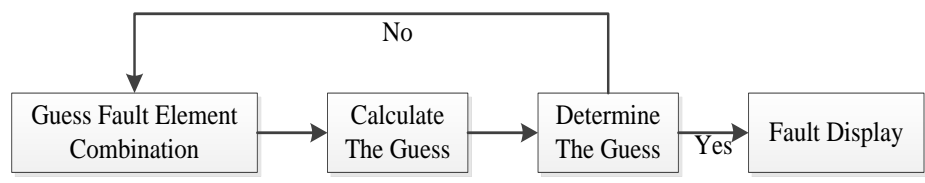

Figure 3 The work flow chart of fault verification method

\section{Modern Methods of Analog Circuit Fault Diagnosis}

Neural Network Fault Diagnosis Methods. Neural network can be achieved on the basic idea is to use the physical device, system or existing computer to simulate the brain structure and function of the artificial system. It is a widely connected giant system, and can be distributed storage information, with functions of parallel processing and self-learning, self-organization and adaptive function. Neural network technology is especially suitable for dealing with the fault diagnosis can't expressed in explicit formula with complex nonlinear relations, and can well solve the traditional pattern recognition method which is difficult to satisfactorily solved due to the nonlinearity, a feedback loop and the problems caused by poor capacity [5]. The information stored in the form of distribution, using network topology and weight distribution to realize nonlinear mapping, using parallel processing to realize the global nonlinear information conversion from input space to the output space, effectively solves the existing in the fault diagnosis of complex systems 'bottleneck' of fault knowledge acquisition and knowledge reasoning problems such as 'combinatorial explosion'. Its specific application mode mainly includes [6]:

a) By using neural network to produce residual. This is mainly using the system identification ability of the neural network, through produce residual sequence identification model, fault detection and diagnosis for the next step

b) By using neural network pattern recognition. For complex systems which is difficult to establish accurate mathematical model, the mathematical model of neural network does not need system, can be implemented only need training data from measure space to fault space mapping so as to identify the system normal mode and fault mode or the difference between different failure modes.

Spain and others apply neural networks to small soft fault diagnosis of analog circuits, with white noise as a test signal source, the failure mode is deviating from the element normal $50 \%$, through neural network for fault classification and fault dictionary query automatically, the result is not only high diagnostic accuracy, and due to the robustness of the neural network, be able to identify without the soft fault modes of learning. 
Aminian studied a multi-layer forward neural network based on the actual circuit fault diagnosis method. The experimental results show that using this method has good robustness of fault diagnosis and the fault diagnosis accuracy of up to $95 \%$.

Catelani apply the radial basis function network to the linear and nonlinear circuit soft fault diagnosis, the experimental results show that the trained network not only for the subsystem or component level failure has good diagnostic capacity, even if the face is not contained in the failure diagnose the new fault can also be successful in the dictionary. Then they apply fuzzy diagnosis method and the radial basis function network diagnosis method to fault diagnosis of analog circuits, the results show that in the presence of noise and the fault component tolerance under the condition of the two fault rate is very low.

The fault diagnosis method based on neural network has many advantages, but based on the inherent mechanism of the neural network is also inevitable exist deficiencies ${ }^{[7]}$ :

a) Using only some examples of definite fault data samples, failed to make full use of the fault diagnosis knowledge of experts in specific areas

b) The learning samples there are some difficulties. Neural network fault diagnosis is built on the basis of a large number of fault data samples; the diagnosis performance is the number of the selected samples and its limit distribution.

c) Not intuitive knowledge expression, diagnosis has a 'black box' behavior, the diagnosis reasoning process difficult to explain.

Fault diagnosis for complex system, due to various types of data need to deal with, can lead to problems such as network scale and learning time is too long, which reduces the neural network is practical.

Theory of Fuzzy Fault Diagnosis Method. Fault diagnosis is through the research to the relationship between the fault symptom and the smell of the system state, because of language expression, and incomplete information of fuzzy attributes are often appeared in the description of the fault symptoms, The relationship between the fault and symptom is difficult to use accurate mathematical model of the said, lead to some fault status is fuzzy [8]. Fuzzy logic provides expression and processing mechanism of the concept of fuzzy logic, the introduction of fuzzy logic can be overcome because of the uncertainty of the complex system itself and the noise of the impact of, so in the complex system fault diagnosis shows certain superiority. Fuzzy fault diagnosis method is to use the membership function of fuzzy mathematics and fuzzy relationship matrix to solve the uncertain relationship between the fault and symptom. The essence of which is to introduce the concept of membership function, the traditional 0 and 1 of binary logic is transferred to a continuum of values on $[0,1]$ interval logic ${ }^{[9]}$.

Bilski Put forward a paper propose decision-making tree simulation system based on fuzzy logic automatic fault diagnosis methods; The method is capable of detecting and locating various faults in the presence of noise. General architecture of the method is also given in this paper, and introduces the noise reduction processing, feature extraction and fault detection. Then the author through the three kinds of physical properties of different system of this method was tested and validated, the results proved the superiority of this method [10].

Carelani proposed a fuzzy automatic diagnosis system based on frequency domain fault dictionary, and through two examples of analog circuit single soft fault analysis of the performance of the proposed method [11].

Pouts proposed a fault dictionary information as the membership function of input variables, and the shape of membership function is determined by the element parameter values in fuzzy diagnosis system, the method is compared with the fault dictionary have greatly improve performance [12].

Fuzzy variable said readable, fuzzy logic reasoning is rigorous, similar to human thinking process, easy to understand. But the system fault diagnosis for complex circuit exists under the principle of selection, the determination of membership function and fuzzy relationship matrix structure, technical 
difficulties. Determine the membership function of not only need knowledge of experience and it takes a long time, the structure of the fuzzy relationship matrix $\mathrm{R}$ need on the basis of a large number of actual operational data, the precision of height depends on the accuracy of the observation data and richness [13].

Kernel Fault Diagnosis Methods. Kernel method is currently one of the most active research directions in the field of machine learning; it is based on statistical learning theory and technology. The kernel function $\mathrm{k}(\mathrm{x}, \mathrm{z})$ is to calculate the two data points under the nonlinear transform $\Phi($.$) image of$ the inner product of the $\mathrm{k}(\mathrm{x}, \mathrm{z})<=\Phi(\mathrm{x}), \Phi(\mathrm{z})$, the $\Phi: \mathrm{x} \rightarrow \Phi(\mathrm{x})$ as the kernel function $\mathrm{k}(\mathrm{x}, \mathrm{z})$ export characteristics of transformation, $x$ as the input space, $(x)$ is a feature space. $K(x, z)$ is defined as a Hilbert space of the inner product, it should first is symmetrical, secondly to satisfy the Mercer condition.

Nuclear method can realize nonlinear transform from space data to feature space, adopt different kernel functions can meet the requirements of different nonlinear transformation. Nuclear method of computation has nothing to do with the dimensions of the feature space, the kernel function is introduced to replace the inner product of the feature space, and export a related to sample, has nothing to do with the sample dimension optimization problem, to avoid the dimension disaster, make accounting method has larger hypothesis space, improve the ability of pattern classification and regression [14]. Data to feature space by kernel function mapping constructs the nuclear matrix, after learning algorithms of processing used in classification mode function.

Methods usually can be divided into supervised kernel method and the unsupervised kernel method two kinds big. In supervised kernel methods, Support Vector Machine is the most typical and is one of the most research and application. Commonly used unsupervised Kernel methods have Kernel Principal Component Analysis, Kernel Clustering, and Kernel Independent Component Analysis etc [15].

Jiang and others will be an improved support vector machine classification is used in the actual fault diagnosis of analog circuits, the results show that this method is compared with the BP network and conventional SVM method has some improve diagnostic accuracy. Wang and others through the optimal wavelet packet transform to extract fault features, uses three different binary tree support vector machine for analog circuit fault diagnosis, the diagnostic accuracy is above $90 \%$. Xiao and others apply the kernel principal component analysis method to simulated circuit fault feature dimension reduction of obtaining the optimal fault features, the experimental results show that the effectiveness of the method [16].

\section{Conclusion}

At present, fuzzy theory, neural network and combined closely with the fault diagnosis of analog circuit fault diagnosis technology is a new development. In recent years, people are increasingly using the emerging theory of fuzzy mathematics and neural network diagnosis knowledge expression of the solution. Widely used knowledge representation methods, which can be easily to knowledge organization into knowledge base form of the computer which can be recognized [17]. The emergence of neural network technology provides a new method for fault diagnosis, especially for those who are due to the fault diagnosis method of analytical model is difficult to establish a system model of complex system, fault diagnosis method based on knowledge is very important, and practical solutions, and has set up a lot in the actual has been successfully used in the system [18]. Analog circuit fault diagnosis technology in recent years there have been a breakthrough development, innovation, in practice there are also many instances of success, but with the rapid development of science and technology, especially the emerging large-scale circuit, analog circuit fault diagnosis is not yet fully realized automation and intellectualization [19-21]. The research field of analog circuit and faced with new challenges, to carry out the study on the method of fault diagnosis theory appears more important. 
And now adays, data driven[22-23] are focused on, this idea is growing in analog circuit diagnosis field.

\section{Acknowledgements}

This work is supported by National Nature Science Foundation under Grant (Project No.61304149, 61573071), the National Natural Science Foundation under Liaoning (Project No.2015020042) and the Excellent Talents to Support Projects in Liaoning Province (Project No.LJQ2015003).

\section{References}

[1] S. Yin, X. Li, H. , O. Kaynak: Data-based techniques focused on modern industry: An overview, IEEE Transactions on Industrial Electronics, Vol.62, No.1 (2015), p.657.

[2] S. Yin, Z. Huang: Performance monitoring for vehicle suspension system via fuzzy positivistic C-means clustering based on accelerometer measurements, IEEE/ASME Transactions on Mechatronics, Vol.20, No.5 (2015), p.2613.

[3] S. Yin, X. Zhu, O. Kaynak, Improved PLS focused on key performance indictor related fault diagnosis, IEEE Transactions on Industrial Electronics, Vol.62, No.3 (2015), p.1651.

[4] S.Y. Yang: Fault diagnosis and reliability of the simulation system design (Tsinghai university press, China1993).

[5] Z.H. Gao: Electronic circuit fault diagnosis technology (Xi 'an university of electronic science and technology press, China 2000), p.1.

[6] S.J. Chen, C.R. Hong and K.F. Wang: Electronic journals, Vol. 28 (2000) No.2, p.127.

[7] M.F. Peng and Y.G. He: Journal of human university: natural science32(2005) No.1, p.25.

[8] M.F. Peng, Y.G. He and M.E. Shen: Journal of, Vol. 21 (2006) No.3, p.118.

[9] W. Zhang, A.Q. Xu and Z.L.Chen: Journal of electronic measurement and instrument, Vol. 20 (2006) No.4, p.46.

[10]P. Wang and S.Y. Yang: IEEE Trans. on CAS I: Regular Papers, Vol. 52 (2005)No.10, p.2118.

[11]P. Wang and S.Y. Yang: Journal of Tsinghai university: natural science edition, Vol. 47 (2007) No.7, p.1245.

[12]M. Hu, H. Wang and G. Hu: Tsinghai Science and Technology,Vol. 12 (2007)No.S1, p.26.

[13]P. Wang and S.Y. Yang: IEEE Tran. OnCASI: Regular Papers, Vol. 52 (2005) No.10, p.2118.

[14]M. Catelani and A. Fort: IEEE Trans.In-strum.Meas, Vol 51 (2002) No.2, p.196.

[15]G. Peng, M.F. Peng and Y.G. He: Microelectronics and computer, Vol. 23 (2006) No.8, p.1.

[16]G.C. Ling, Y.G. He and Y.Q. Zhu: Journal of circuits and systems, Vol. 9 (2004) No.2, p.54.

[17]S. Yin, X. Zhu: Intelligent particle filter and its application on fault detection of nonlinear system, IEEE Transactions on Industrial Electronics, Vol.62, No.6 (2015),p.3852.

[18] S. Yin, O. Kaynak: Big data for modern industry: challenges and trends, Proceedings of the IEEE, Vol.102, No.3(2015), p.143 